\title{
A Dynamic Test to Identify the Potential Recess Between the Psoas Major and Quadratus Lumborum Muscles at the Supra-lliac Level
}

\author{
Huili Li (D) \\ Rong Shi \\ Yun Wang (1D)
}

Department of Anesthesiology, Beijing Chaoyang Hospital, Capital Medical University, Beijing, 100020, People's Republic of China
Correspondence: Yun Wang

Department of Anesthesiology, Beijing

Chaoyang Hospital, Capital Medical

University, Beijing, 100020, People's

Republic of China

Email wangyun129@ccmu.edu.cn
Purpose: The transmuscular quadratus lumborum block (TQLB) deposits the local anesthetic into the fascial interspace between the quadratus lumborum (QL) and psoas major (PM) muscles in the lumbar paravertebral regions. However, there are multiple anatomical relationships between the QL and PM muscles in the cross section of ultrasound scanning at the supra-iliac level, which may influence the identification of injection site with the TQLB. In the current study, we developed a dynamic test to identify the anatomical relationships between the QL and PM muscles.

Methods: Forty male and 40 female volunteers were enrolled from June 20, 2021 to July 2, 2021 at Beijing Chaoyang Hospital, Capital Medical University for this descriptive study. The ultrasound identification of the cross-section anatomical relationship between the QL and PM muscles was performed under the shamrock sonogram at the supra-iliac level. The types of anatomical relationship between the QL and PM muscles were divided into "separate" or "non-separate". The volunteers who were identified as "non-separate" received the dynamic tests. The numbers of positive dynamic tests in "non-separate" volunteers were recorded.

Results: Six (15\%) male volunteers and 10 (25\%) female volunteers were identified as "separate". Thirty-four male and 30 female volunteers who were identified as "non-separate" received the dynamic tests. The positive dynamic tests were observed in $8(23.5 \%)$ male and 9 (30\%) female volunteers, respectively.

Conclusion: Our study showed that the potential recess between the encased QL and encased PM muscles may exist in "non-separate" subjects, in which the local anesthetic is injected may result in the block failure. A dynamic test could help distinguish the potential recess.

Keywords: posture test, ultrasound guidance, quadratus lumborum block

\section{Introduction}

Laterally, the QL muscles fill the space between ribs XII and the iliac crest on both sides of the vertebral column. Cross-section of the posterior abdominal wall shows the QL muscles are typically overlapped medially by the PM muscles. The overlapping QL and PM muscles split approximately at the level of L4, since the QL muscle travels laterally and inserts on the ilium, while the PM muscle travels laterally and downward and inserts on the lesser trochanter (Figure 1A and B). At the cranial level of the split between the QL and PM muscles, the transversalis fascia (TF) covers the abdominal surface of both QL and PM muscles (Figure 1A). ${ }^{1}$ 

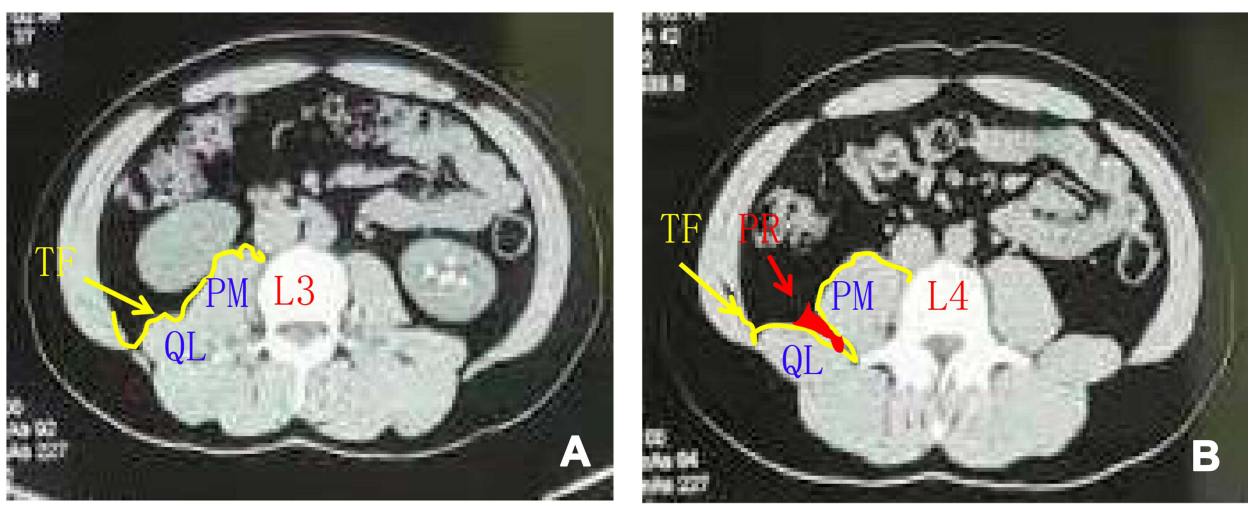

Figure I The anatomical relationship between the QL and PM muscles at the cross-sections of CT images. (A) The cross-section of L3 TP shows the overlap between the $\mathrm{QL}$ and PM muscle. The TF covers the abdominal surface of both QL and PM muscles. (B) The cross-section of L4 TP shows the separation (bifurcation) of the QL and PM muscles. The PR occurs between the QL and PM muscles.

Abbreviations: QL, quadratus lumborum; PM, psoas major; CT, computerized tomography; TP, transverse process; PR, potential recess.

However, at the caudal level of the split, the TF encases the QL and PM muscles, respectively (Figure 1B).

Dr. Borglum et al proposed the TQLB in 2013, which is widely used in postoperative analgesia for abdominal or hip surgery. ${ }^{2}$ The TQLB deposits the local anesthetic (LA) into the fascial interspace between the QL and PM muscles in the lumbar paravertebral regions. ${ }^{3}$ In fact, the action mechanism of the TQLB is associated with the anatomy of the TF. At the level of the arcuate ligaments of the diaphragm, the TF splits into two layers. One layer is continuous with the endothoracic fascia, and another layer becomes the inferior diaphragmatic fascia. Hence, the spread of the injectate from the lumbar injection site into the lower thoracic paravertebral space via the medial and lateral arcuate ligaments with the TQLB is posterior to the TF.

However, based on the above anatomical analysis, there are three anatomical relationships between the QL and PM muscles in the cross section under ultrasound scanning: overlap of QL and PM muscles, contact of TF-encased-QL and TF-encased-PM muscles, and separation of TF-encased-QL and TF-encased-PM muscles. Furthermore, it is difficult to distinguish the overlap of QL and PM muscles from the contact of encased-QL and encased-PM muscles under ultrasonography, since the point-of-care ultrasound has a lower resolution for the fascia imaging. Therefore, it is possible to inject the local anesthetic into the potential recess between the TF-encased-QL and TF-encased-PM muscles if the relative anatomy was not correctly identified. In the current study, we hypothesize that the dynamic test developed could be used to identify the anatomical relationships between the QL and PM muscles in the cross section of ultrasound scanning, and as a consequence optimize the injection site.

\section{Methods}

Ethical approval for our study protocol was provided by the Ethics Committee of Beijing Chaoyang Hospital, Capital Medical University, China on June 16, 2021 with reference number 2021-532. This study was conducted in accordance with the Declaration of Helsinki. After the written informed consent was obtained, 80 volunteers were enrolled from June 20, 2021 - July 2, 2021 at Beijing Chaoyang Hospital, Capital Medical University for this descriptive study. Patients were excluded if they had a body mass index $(\mathrm{BMI})>30$ and more than 70 years, clinically known spinal deformity, infection in the back, allergy to LA, or coagulopathy.

After the patient arrived in the operating theatre, intravenous access was established, and patient vitals including heart rate, arterial pressure, arterial oxygen saturation, and electrocardiography were monitored. The patients were positioned in the lateral decubitus position with the legs straight. All ultrasound scans were performed using an ultrasound system and a curved array probe (C5-2, 5-2 MHz frequency, KONICA MINOLTA, Tokyo, Japan). The transducer is placed in the transverse position just cranial to the iliac crest at the level of the posterior axillary line. ${ }^{4}$ With the PM muscle anteriorly, the erector spinae muscle posteriorly and the QL muscle adherent to the apex of the transverse process, a well-recognizable pattern of a shamrock with three leaves can be seen (Figure 2A). Then, the anatomical relationship between the QL and PM muscles in the cross section was 

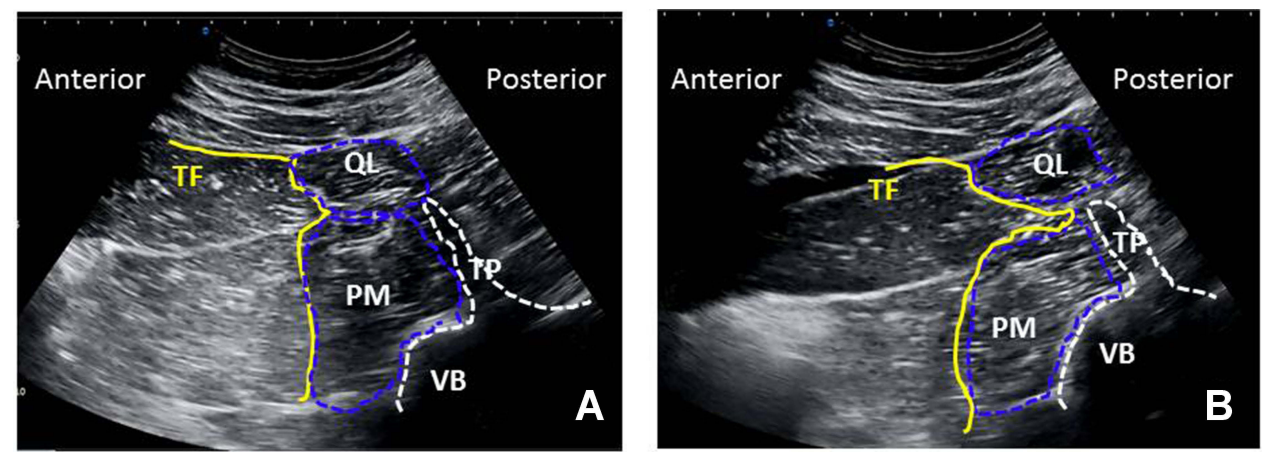

Figure 2 The shamrock signs of sonograms before and after the dynamic test with the transducer in the transverse position just cranial to the iliac crest. (A) The shamrock sonogram shows the non-separation between the QL muscle and PM muscle in a patient with the lateral decubitus position and the legs straight. (B) The patient is asked to flex the knees and hips toward the abdomen and the sonogram shows that the fat in the preperitoneal space is crushed into the potential recess between the QL and PM muscles.

Abbreviations: QL, quadratus lumborum; PM, psoas major; TP, transverse process; VB, vertebral body.

identified on the sonogram. All volunteers were identified as "separate" or "non-separate" by an independent expert experienced in ultrasound imaging. Then, the volunteers who were identified as "non-separate" were subject to a dynamic test. The dynamic test was designed to identify the anatomical relationship between the QL and PM muscles in "non-separate" subjects. In detail, the volunteer was asked to flex the knees and hips toward the abdomen, while the transducer was kept intact. The dynamic test was considered as positive if the fat in the preperitoneal space was crushed into the potential recess between the TF-encased QL and TFencased PM muscles (Figures 2A and B, Video). The positive dynamic test indicates the anatomical type as the contact of the TF-encased QL and TF-encased PM muscles.

\section{Results}

No subjects dropped out in the study. We recorded data from 40 male (mean age $31.4 \pm 5.6$ years; mean BMI 29.2 $\pm 4.3 \mathrm{~kg} \mathrm{~m}^{-2}$ ) and 40 female (mean age $29.8 \pm 6.2$ years; mean BMI $28.7 \pm 5.4 \mathrm{~kg} \mathrm{~m}^{-2}$ ) volunteers. For the ultrasound identification of the cross-section anatomical relationship between the QL and PM muscles, 6 (15\%) male volunteers and $10(25 \%)$ female volunteers were identified as "separate". The 34 male and 30 female volunteers who were identified as "non-separate" were conducted with the dynamic tests. The positive dynamic tests were observed in $8(23.5 \%)$ male, and $9(30 \%)$ female volunteers, respectively.

\section{Discussion}

Our study showed that a dynamic test could help identify the preperitoneal recess between the TF-encased QL and TF-encased PM muscles, which may avoid the wrong injection into the preperitoneal space when performing the TQLB at the supra-iliac level.

The TQLB is usually performed on patients in the lateral decubitus position using the shamrock sign as the internal sonographic landmark. ${ }^{3}$ Notably, with the TQLB, the endpoint of needle tip lies at the cranial, but not caudal level of split between the QL and PM muscles. It is very important to distinguish the split level of the QL and PM muscles under the sonogram, since the contact between the TF-encased QL muscle and TF-encased PM muscle below the split level still can exist. Otherwise, the LA can be wrongly injected into the potential recess between the TFencased QL muscle and TF-encased PM muscle below the split level when the TQLB is performed. The correct LA deposit with the TQLB should be into the fascial interspace between the QL and PM muscles and allows the LA to spread cranially to enter the lower thoracic paravertebral space posterior to the TF. The wrong injection into the potential recess between the TF-encased QL muscle and TF-encased PM muscle may result in the block failure.

The dynamic test we developed can be used to identify the relative anatomical relationship between the QL and PM muscles. This posture can induce the contraction of PM muscle and increase the abdominal pressure. On the sonogram, it could be visualized that the contraction of PM muscle trends to make itself to leave the QL muscle and the fat in the preperitoneal space was crushed into the potential recess between the QL and PM muscles (Figure 2B and Video).

In the current study, we included 40 male and 40 female, since the high iliac crest, which is common in male, may influence the split level of QL and PM muscles. Our data showed that $25 \%$ female were identified as 
"separate", which is higher than that $(15 \%)$ in male. It may be associated with the gender difference in high iliac crest. The positive dynamic tests were observed in $23.5 \%$ male, and $30 \%$ female volunteers, respectively. It indicates that the potential recess between the encased QL and encased PM muscles may exist in "non-separate" subjects, in which the local anesthetic is injected may result in the block failure. Therefore, it is very important to routinely use the dynamic test to identify the anatomical relationship between the QL and PM muscles when performing the TQLB at the supra-iliac level.

The current study has several limitations. First, we only included the patients with BMI $<30$. Future studies should evaluate the utility of the test we described above in the obese patients. Another limitation of our study is that it is a descriptive, but not randomized trial. Larger randomized trials are required to quantify the reliability and validity of the test described in the future.

\section{Conclusions}

In summary, we suggest that the dynamic test should be performed to identify the potential recess of the TFencased QL and TF-encased PM muscles when the TQLB is performed just cranially to the iliac crest. Furthermore, larger randomized trials are required to quantify the reliability and validity of the test described in the future.

\section{Acknowledgments}

This work was supported by the Beijing Hospitals Authority Clinical medicine Development of special funding support (XMLX202106).

\section{Disclosure}

The authors declare no conflicts of interest.

\section{References}

1. Elsharkawy H, El-Boghdadly K, Barrington M. Quadratus lumborum block: anatomical concepts, mechanisms, and techniques. Anesthesiology. 2019;130(2):322-335. doi:10.1097/ALN.0000000000002524

2. Børglum J, Jensen K, Moriggl B, L€onnqvist P, Christensen A, Sauter A. Ultrasound-guided transmuscular quadratus lumborum blockade. Br J Anaesth. 2013. Available from: http://bja.oxfordjour nals.org/forum/topic/brjana_el\%3B9919. Accessed November 30, 2015.

3. Dam M, Moriggl B, Hansen CK, Hoermann R, Bendtsen TF, Børglum J. The pathway of injectate spread with the transmuscular quadratus lumborum block: a Cadaver Study. Anesth Analg. 2017;125 (1):303-312.3. doi:10.1213/ANE.0000000000001922

4. Dam M, Hansen C, Poulsen TD, et al. Transmuscular quadratus lumborum block reduces opioid consumption and prolongs time to first opioid demand after laparoscopic nephrectomy. Reg Anesth Pain Med. 2021;46(1):18-24. doi:10.1136/rapm-2020-101745
Journal of Pain Research

\section{Publish your work in this journal}

The Journal of Pain Research is an international, peer reviewed, open access, online journal that welcomes laboratory and clinical findings in the fields of pain research and the prevention and management of pain. Original research, reviews, symposium reports, hypothesis formation and commentaries are all considered for publication. The manuscript management system is completely online and includes a very quick and fair peer-review system, which is all easy to use. Visit http:// www.dovepress.com/testimonials.php to read real quotes from published authors. 